Michigan State University

National Superconducting Cyclotron Laboratory

EXCITED STATE POPULATIONS FOR EQUILIBRIUM AND PREEQUILIBRIUM EMISSION

F. ZHU, M.J. HUANG, W.G. LYNCH, T. MURAKAMI, Y.D. KIM, T.K. NAYAK, R. PELAK, M.B. TSANG, H.M. XU, W.G. GONG, K. KWIATKOWSKI, R. PLANETA, S. ROSE, V.E. VIOLA, Jr., L.W. WOO, S. YENNELLO, and J. ZHANG



MSUCL-912 



\title{
Excited State Populations for Equilibrium and Preequilibrium Emission
}

\author{
F. Zhu' ${ }^{a}$, M.J. Huang, W.G. Lynch, T. Murakamib , Y.D. Kim , T.K. Nayak ${ }^{d}$, R. Pelake, M.B. \\ Tsang, H.M. Xú ${ }^{\mathfrak{f}}$, and W.G. Gongg, \\ Department of Physics and Astronomy and National Superconducting Cyclotron Laboratory \\ Michigan State University, East Lansing, Michigan 48824.
}

K. Kwiatkowski, R. Planetah , S. Rose , V.E. Viola, Jr., L.W. Wooj, S. Yennellof, and J. Zhangk, Indiana University Cyclotron Facility Indiana University, Bloomington, Indiana 47405

\begin{abstract}
Populations of excited ${ }^{5} \mathrm{Li}$ and ${ }^{6} \mathrm{Li}$ fragments have been measured for the ${ }^{3} \mathrm{He}+\mathrm{Ag}$ reaction at $200 \mathrm{MeV}$. At forward angles, fragments emitted via preequilibrium mechanisms display population probabilities that are consistent with emission temperatures of about 4 $\mathrm{MeV}$, comparable to other measurements of preequilibrium processes. In contrast, fragments emitted to backward angles display much smaller population probabilities consistent with emission temperatures of about $1 \mathrm{MeV}$. Such low temperatures are consistent with residue excitation energies predicted by molecular dynamics calculations.
\end{abstract}

PPACS index: $25.70 \mathrm{~Np}$

\footnotetext{
a Present Address: Brookhaven National Laboratory, Upton NY, 11973.

b Present Address: Kyoto University, Kyoto 606, Japan.

c Present Address: Department of Physics, KeK, Ibaraki 305, Japan.

d Present Address: Columbia University Nevis Labs, Irvington NY, 10533.

e Present Address: Comell University, Ithaca NY, 14853.

f Present Address: Texas A\&M University, College Station TX, 77843.

8 Present Address: Lawrence Berkeley Laboratory, Berkeley CA, 94720.

h Present Address: Jagellonian University, Krakow, Poland.

i Present Address: Iowa State University, Ames IA 50011.

j Present Address: Sabbagh Associates, Bloomington IN, 47401.

k Present Address: Institute for Atomic Energy, Beijing, PRC.
} 
In general, the emission of intermediate mass fragments (IMF's: $Z=3-20$ ) occurs via a mixture of preequilibrium and equilibrium emission mechanisms[1-4]. These two mechanisms can be better distinguished for light ion than for heavy ion induced reactions due to the rather small momenta carried by light ion beams and the absence of deep inelastic scattering phenomena. The relative importance of preequilibrium emission increases with incident energy[2,4-6]. For example, compound nuclear emission mechanisms account for most of the fragments emitted in ${ }^{3} \mathrm{He}$ induced reactions at low incident energies, $E \leq 60 \mathrm{MeV}[5]$; at $E \approx 200 \mathrm{MeV}$, the two contributions become comparable[2].

While statistical models for compound nuclear decay have been fairly successful in describing the emission of fragments from the compound nucleus[2,5,6], a comparable understanding of preequilibrium fragment emission in light ion induced reactions has been elusive. Dynamical models $[7,8]$ for fragment emission generally underpredict the yields for preequilibrium fragment emission[9-11]. On the other hand, some success in describing the fragment yields and energy spectra has been achieved by assuming the statistical emission of IMF's from a hot thermalized subsystem, formed by the overlap of the projectile with the target, as this subsystem equilibrates with the remainder of the target $[1,2]$.

Integral to the success of this latter model[1] is the assumption that the subsystem has a local temperature which is significantly larger than the temperature of the compound nucleus. Such a local thermalization, however, should also be reflected by differences between the emission temperatures[12-15] extracted from the decays of excited IMF's emitted by preequilibrium processes to forward angles and those extracted from equilibrium emissions to backward angles. For example, emission temperatures of about 6.0 and $2.8 \mathrm{MeV}$ are predicted via the accreting source model of ref. [1] for ${ }^{6} \mathrm{Li}$ fragments emitted to forward and backward angles, respectively, in the ${ }^{3} \mathrm{He}+\mathrm{Ag}$ reaction at $\mathrm{E}_{\mathrm{lab}}=200$ $\mathrm{MeV}$. In this work, we describe measurements performed to test for such manifestations of a local thermalization of the incident collective energy.

The experiment was performed by bombarding a natural $1.05 \mathrm{mg} / \mathrm{cm}^{2} \mathrm{Ag}$ target by a $200 \mathrm{MeV}^{3} \mathrm{He}$ beam from the Indiana University Cyclotron Facility. Coincident light particles were detected with a high resolution hodoscope[12] consisting of 13 closely packed telescopes. Four of these telescopes were each comprised of four silicon detectors ( $75 \mu \mathrm{m}, 100 \mu \mathrm{m}, 5 \mathrm{~mm}$, and $5 \mathrm{~mm}$ thick). The other nine telescopes were each comprised of two silicon detectors ( $200 \mu \mathrm{m}$ and $5 \mathrm{~mm}$ thick) and a $10 \mathrm{~cm}$ thick $\mathrm{NaI}(\mathrm{Tl})$ detector. Two single-wire gas counters were placed in front of each telescope to provide $x$ and $y$ position information. Energy calibrations of the silicon detectors were obtained using an ${ }^{241} \mathrm{Am}$ 
source and extended to higher energies with a precision pulser. The $\mathrm{NaI}(\mathrm{Tl})$ detectors were calibrated with the $\Delta \mathrm{E}$ information from the $5 \mathrm{~mm} \mathrm{Si}(\mathrm{Li})$ detectors and by detecting recoil protons produced in the bombardment of a polypropylene target by $200 \mathrm{MeV} 3 \mathrm{He}$ ions. Measurements were performed with the hodoscope centered at $\Theta_{\text {lab }}=42^{\circ}$ and $109^{\circ}$.

Energy spectra for this reaction [2] are consistent with the emission of fragments predominantly via preequilibrium decay processes at forward angles and via equilibrium decay processes at backward angles. The excited state populations in $5 \mathrm{Li}$ and 6 Li nuclei relevant for this work can be easily observed via correlation functions, $R\left(E_{\text {rel }}\right)$, defined in terms of the measured coincidence yield $Y_{12}\left(p_{1}, p_{2}\right)$ and the singles yields $Y_{1}\left(p_{1}\right)$ and $\mathrm{Y}_{2}\left(\mathrm{p}_{2}\right)$ :

$$
\Sigma Y_{12}\left(\mathrm{p}_{1}, \mathrm{p}_{2}\right)=\mathrm{C}\left[1+\mathrm{R}\left(\mathrm{E}_{\mathrm{rel}}\right)\right] \cdot \Sigma \mathrm{Y}_{1}\left(\mathrm{p}_{1}\right) \mathrm{Y}_{2}\left(\mathrm{p}_{2}\right)
$$

Here, $\mathbf{p}_{1}$ and $\mathbf{p}_{2}$ are the momenta of the two particles in the laboratory and $E_{r e l}$ is the relative energy between the two particles. The sums on both sides of Eq. 1 are extended over all energy, position and detector combinations corresponding to specific relative energy bins. The normalization constant $C$ in $E q . ~(1)$ is chosen so that $1+R\left(E_{r e l}\right)$ is unity for values of $E_{r e l}$, significantly above the channel threshold where resonances are not observed in the exit channel.

Figure 1 shows the $d-3 \mathrm{He}, \mathrm{p}-\alpha$, and $d-\alpha$ correlation functions measured at $\Theta_{\mathrm{lab}}=42^{\circ}$. The narrow peak in the $\mathrm{d}-3 \mathrm{He}$ correlation function (upper panel) corresponds to the $E^{*}=16.7 \mathrm{MeV}$ excited state of ${ }^{5} \mathrm{Li}$; details are given in Table 1 . The $\mathrm{p}-\alpha$ correlation function (middle panel) reveals the comparatively broad ground state of $5 \mathrm{Li}$. The narrow peak in the $\mathrm{d}-\alpha$ correlation function (lower panel) at $\mathrm{E}_{\mathrm{rel}}=0.71 \mathrm{MeV}$ and the broad structure at $\mathrm{E}_{\mathrm{rel}}=$ $2.8 \mathrm{MeV}$ correspond to the $E^{*}=2.19 \mathrm{MeV}$ excited state and the overlapping $E^{*}=4.31$ and $E^{*}=5.65$ excited states of $6 \mathrm{Li}$, respectively. Figure 2 shows the $d$ - $\alpha$ correlation function for $\Theta$ lab $=109^{\circ}$. Here, the $E^{*}=2.19 \mathrm{MeV}$ excited state is easily observed, but the higher lying states at $E^{*}=4.31$ and $5.65 \mathrm{MeV}$ are not, and the yields of $5 \mathrm{Li}$ nuclei at $\Theta_{\text {lab }}=109^{\circ}$ (not shown) are also too small to be clearly observed. These latter observations are consistent with a low emission temperature at $\Theta_{\text {lab }}=109^{\circ}$. The suppression of proton-rich ${ }^{5} \mathrm{Li}$ emission from the more neutron-rich composite system could also be a factor[16].

Following ref. [13], the yields of these particle unstable states were obtained by fitting the measured yields corresponding to the peaks in Figs. 1 and 2 with resonance line shapes, while correcting for detection efficiencies and non-resonant combinatorial backgrounds[12]. Reasonable fits were obtained for backgrounds lying within the values bounded by the dashed lines in Figs. 1 and 2; the best fits are shown by the solid curves. The population probability for each state is obtained by dividing by the $(2 J+1)$ spin 
degeneracy and by the yield of stable ${ }^{6} \mathrm{Li}$ nuclei[13]. Unresolved ${ }^{6} \mathrm{Li}$ states at $E^{*}=4.31$ and $5.65 \mathrm{MeV}$ were assigned the same population probability within the fits.

In most statistical models[17], the initial excited state population probabilities are assumed to be proportional to $\exp \left(-E^{*} / T_{e m}\right)$ where $T_{e m}$ is the temperature of the system when the fragment is emitted. Modifications to the initial populations of excited states caused by the sequential feeding from heavier particle unstable nuclei have been estimated via sequential decay calculations. In these calculations, the excited states of primary emitted fragments are populated thermally, and then allowed to decay, using approximations outlined in ref. [13]. Unknown spins or parities of low lying discrete states were assigned randomly and the calculations were repeated to assess the sensitivities of the population probabilities to these spectroscopic uncertainties.

The dotted-dashed curves in Fig. 3 show: (panel a) the predictions for the ratio of the initial population probabilities (before sequential feeding) for the two measured particle unstable states of ${ }^{5} \mathrm{Li}$, (panel b) the population probability for the $E^{*}=2.19 \mathrm{MeV}$ excited state of ${ }^{6} \mathrm{Li}$, (panel $\mathrm{c}$ ) the average population probability for the $\mathrm{E}^{*}=4.31$ and $5.65 \mathrm{MeV}$ excited states of ${ }^{6} \mathrm{Li}$, and (panel $\mathrm{d}$ ) the ratio of the average population probability for the $E^{*}=4.31$ and $5.65 \mathrm{MeV}$ states to the population probability for the $E^{*}=2.19 \mathrm{MeV}$ excited state of ${ }^{6} \mathrm{Li}$. The solid lines in the figure bound the range of values predicted by the sequential decay calculations for different assumptions about the spins and parities of higher lying particle unstable states which sequentially feed the excited states of interest.

The cross-hatched regions bounded by dashed lines indicate the range of values that are consistent with the measurements at $\Theta_{\mathrm{lab}}=42^{\circ}$. A reasonable overall agreement between calculated and measured values is observed for emission temperatures of about $4 \mathrm{MeV}$; this value is comparable to the temperatures, $3 \mathrm{MeV} \leq \mathrm{T}_{\mathrm{em}} \leq 6 \mathrm{MeV}$, measured for preequilibrium emission in other reactions[15]. In contrast, the dotted lines in Fig. $3 b$ indicate that a much lower population probability, consistent with an emission temperature of about $1 \mathrm{MeV}$, is obtained at $\Theta_{\mathrm{lab}}=109^{\circ}$ for the $E^{*}=2.19 \mathrm{MeV}$ excited state of ${ }^{6} \mathrm{Li}$. While large differences between forward and backward angle emission temperatures are qualitatively consistent with a local thermalization of the incident kinetic energy, both values are much smaller than the values predicted by the accreting source model of ref. [1]. The discrepancy at $\Theta_{\mathrm{lab}}=109^{\circ}$ suggests, in particular, that significantly more energy is radiated via preequilibrium emission than the accreting source model[1] predicts.

The observed emission temperatures are also lower than the temperatures extracted from fits to the IMF energy spectra[2], assuming a preequilibrium source (yielding $T \approx 8$ $\mathrm{MeV}$ ) which dominates at forward angles and an isotropic equilibrated source (yielding $\mathrm{T}=$ 3.4 MeV) which dominates at backward angles. Other investigations[15] have reported 
similar observations and have suggested that spectral temperatures may be enhanced due to nonthermal contributions from collective motion[18], and emission temperatures reduced due to cooling via expansion[19-22] or via preequilibrium emission[19,22] or both. The present work confirms observations[15,23] that emission temperatures can be less than spectral temperatures for angles where equilibrium emission mechanisms are more dominant. This latter inconsistency suggests either difficulties in the theoretical interpretation of emission temperatures for equilibrium decays, or alterations of the energy spectra at backward angles due to rotational effects[24], or contributions from nonequilibrium emission mechanisms[25], or both.

Emission temperatures for preequilibrium processes have been reproduced via transport model calculations[22,26]. It is interesting to explore whether the low emission temperatures of target-like residues observed in this work can be similarly reproduced. The calculation of such low residue temperatures, however, requires numerical accuracies in excitation energy computation that are presently only achieved within the QPD molecular dynamics model[7,26]. Temperatures of daughter residual nuclei after $6_{\mathrm{Li}}$ emission were therefore calculated assuming: (1) first chance emission, (2) a level density parameter of $\mathrm{a}=\mathrm{A} / 8 \mathrm{MeV}^{-1}$, and (3) residue excitation energies and angular momenta provided by the QPD model. The resulting emission temperatures, shown by the solid points in Fig. 4 as a function of impact parameter, were evaluated after an elapsed time of $90 \mathrm{fm} / \mathrm{c}$ and corrected for the separation energy and the measured average kinetic energy taken away by the excited ${ }^{6} \mathrm{Li}$ nuclei. The error bars reflect the theoretical uncertainties in determining the freezeout time at the end of the preequilibrium cascade. For central collisions, $b \leq 2$, the calculations are close to the measured value indicated by the crosshatched region. Due to the large mean kinetic and separation energies of the measured ${ }^{6} \mathrm{Li}$ particle unstable nuclei, the temperature of the parent residual nucleus $(2.5 \mathrm{MeV})$ is significantly higher than that of the daughter nucleus. In this respect, the discrepancy between the calculated temperature of the parent residual nucleus and the spectral temperature of the equilibrium source may be reduced but not eliminated. The present measurements do suggest temperatures that are somewhat low to be consistent with an abundant equilibrium emission of IMF's. Unfortunately, QPD model calculations underpredict the yield of preequilibrium IMF's considerably, therefore comparisons of QPD model calculations, following ref. [26], are not currently feasible.

In summary, populations of excited ${ }^{5} \mathrm{Li}$ and ${ }^{6} \mathrm{Li}$ fragments have been measured for the ${ }^{3} \mathrm{He}+\mathrm{Ag}$ reaction at $200 \mathrm{MeV}$. For fragments emitted to forward angles by noncompound emission mechanisms, emission temperatures of about $4 \mathrm{MeV}$ are observed, similar to other measurements of preequilibrium processes. Fragments emitted 
to backward angles, however, display much smaller populations probabilities consistent with an emission temperature of about $1 \mathrm{MeV}$. This surprisingly low emission temperature at $\Theta_{\text {lab }}=109^{\circ}$ is consistent with the predictions of QPD molecular dynamics model calculations.

This paper is based upon work supported by the National Science Foundation under Grant No. PHY-89-13815 and by the Department of Energy under Grant No. DE.FG0288ER.40404.A000. 


\section{References}

1. D.J. Fields et al, Phys. Rev. C30 (1984) 1912.

2. K. Kwiatkowski et al, Phys. Lett. B 171 (1986) 41.

3. J.L. Wile et al, Phys. Rev C45 (1992) 2300.

4. S.J. Yennello et al, Phys. Lett. B 246 (1990) 46.

5. L.G. Sobotka et al, Phys. Rev. Lett. 51 (1983) 2187.

6. M.A. McMahan et al, Phys. Rev. Lett. 54 (1985) 1995.

7. D.H. Boal and J.N. Glosli, Phys. Rev. C 38 (1988) 1870.

8. G. Peilert et al, Phys. Rev. C46 (1992) 1457.

9. D.R. Bowman et al, Phys. Rev. Lett. 67 (1991) 1527.

10. R.T. de Souza, Phys. Lett. B 268 (1991) 6.

11. M.B.Tsang, Phys. Rev. Lett. 71 (1993) 1502.

12. T. Murakami et al, Nucl. Inst. and Meth. A275 (1989) 112.

13. T.K. Nayak et al, Phys. Rev. C45 (1992) 132.

14. Z. Chen et al, Phys. Rev C36 (1987) 2297.

15. C. Schwarz et al, Phys. Rev. C48 (1993) 676.

16. J. Brzychczyk et al, Phys. Rev. C47 (1993) 1553.

17. See for example: J. Randrup and S.E. Koonin, Nucl. Phys. A356 (1981) 223; D.H.E. Gross et al, Z.Phys. A309, (1982) 41; W.A. Friedman W.G. Lynch, Phys. Rev. C28 (1983) 16; J. Gomez del Campo, Phys. Rev. Lett. 61 (1988) 290.

18. M.B. Tsang et al, Phys. Rev. Lett. 60 (1988) 1479.

19. W.A. Friedman, Phys. Rev. Lett. 60 (1988) 2125.

20. J. Bondorf et al, Nucl. Phys. A443 (1985) 321.

21. D.H.E. Gross et al, Phys. Lett. B 203 (1988) 26.

22. H.M. Xu et al, Phys. Lett. B 299 (1993) 199.

23. H. Dabrowski et al, Phy. Lett. B 247 (1993) 223.

24. C.B. Chitwood et al, Phys. Rev. C34 (1986) 858.

25. W. Skulski et al, Phys. Lett. B 218 (1989) 7. 
26. D.H. Boal et al, Phys. Rev. Lett. 62 (1989) 737. 
Table 1.: Spectroscopic information for excited states of ${ }^{5} \mathrm{Li}$ and ${ }^{6} \mathrm{Li}$ nuclei. The last column provides the branching ratio to the observed decay channel.

\begin{tabular}{|l|l|l|l|l|}
\hline Nucleus & $E^{*}(\mathrm{MeV})$ & $J^{\pi}$ & $\Gamma(\mathrm{MeV})$ & B.R. \\
\hline $5 \mathrm{Li}$ & g.s. & $3 / 2^{-}$ & 1.5 & 1.0 \\
\hline $5 \mathrm{Li}$ & 16.7 & $3 / 2^{+}$ & 0.2 & 0.86 \\
\hline${ }^{6} \mathrm{Li}$ & 2.19 & $3^{+}$ & 0.024 & 1.0 \\
\hline${ }^{6} \mathrm{Li}$ & 4.31 & $2^{+}$ & 1.7 & 0.97 \\
\hline${ }^{6} \mathrm{Li}$ & 5.65 & $1^{+}$ & 1.5 & 0.74 \\
\hline
\end{tabular}




\section{Figure Captions}

Fig.1: Correlation functions measured at $\Theta_{\text {lab }}=42^{\circ}$ for coincident deuterons and ${ }^{3} \mathrm{He}$ particles (upper panel), protons and $\alpha$ particles (center panel), and deuterons and $\alpha$ particles (lower panel). The solid and dashed curves are discussed in the text.

Fig.2: Correlation functions for coincident deuterons and $\alpha$ particles, measured at $\Theta_{\text {lab }}=$ $109^{\circ}$. The solid and dashed curves are discussed in the text.

Fig. 3: Temperature dependence of the calculated ratios of excited state population probabilities for the specific sets of states indicated in the figure and discussed in the text. The various curves and cross-hatched regions are discussed in the text.

Fig. 4: Residue temperatures calculated using the QPD model are depicted for different impact parameters by the solid points. The crossed hatch region depicts the emission temperature observed at $\Theta_{\mathrm{lab}}=109^{\circ}$. 


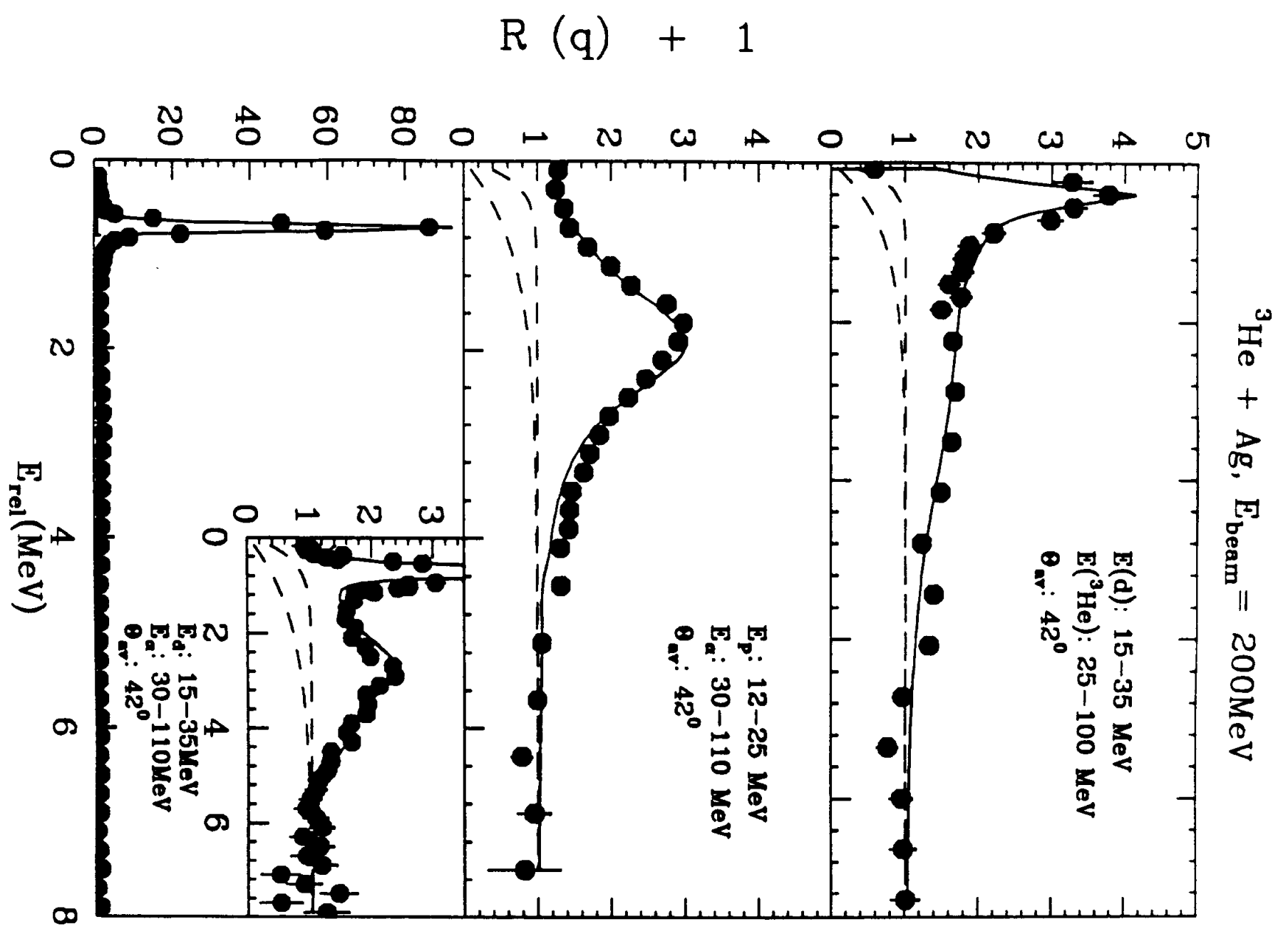

Fig. 1 




Fig. 2 
Ratio

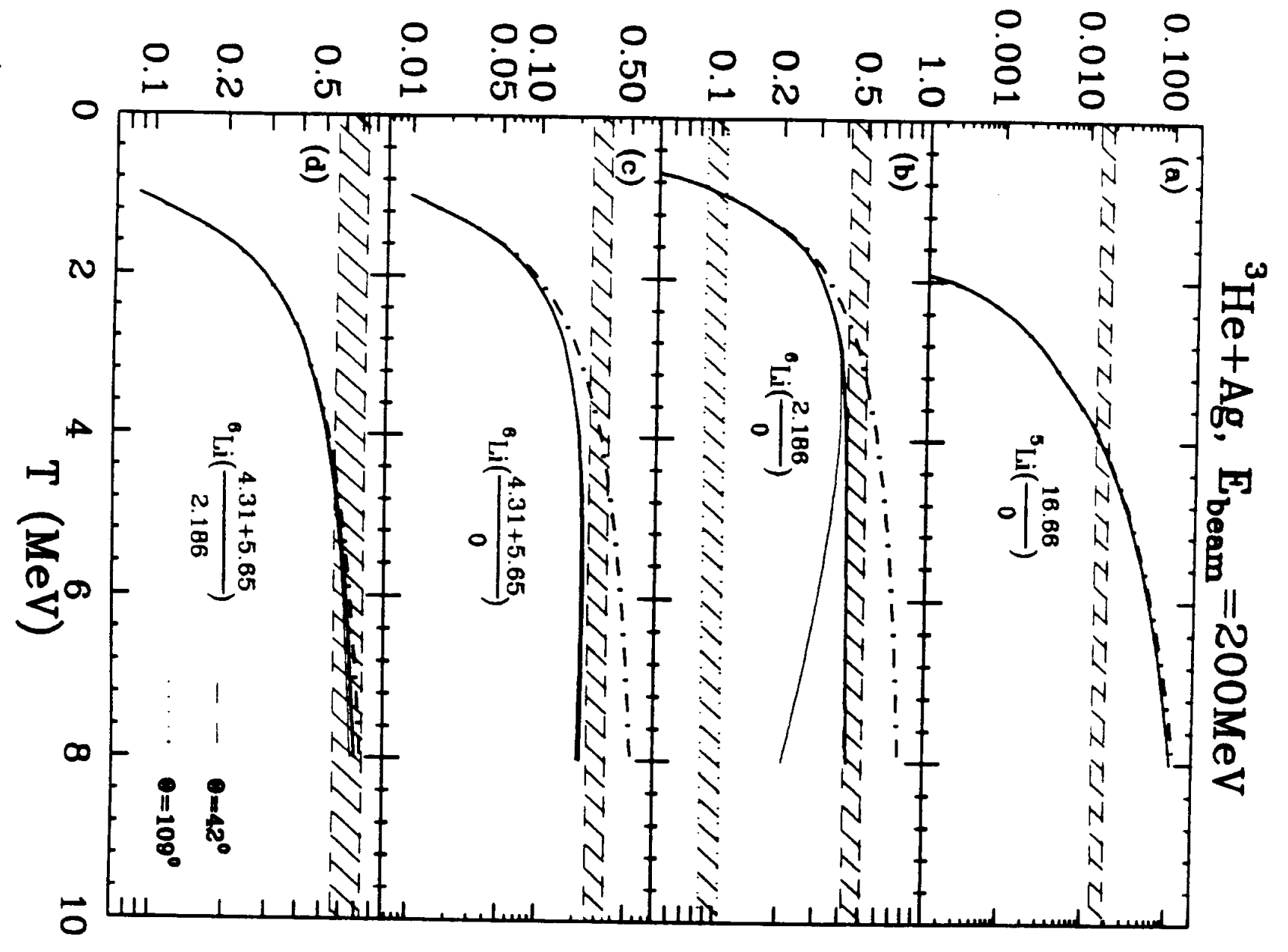

Fig 3 


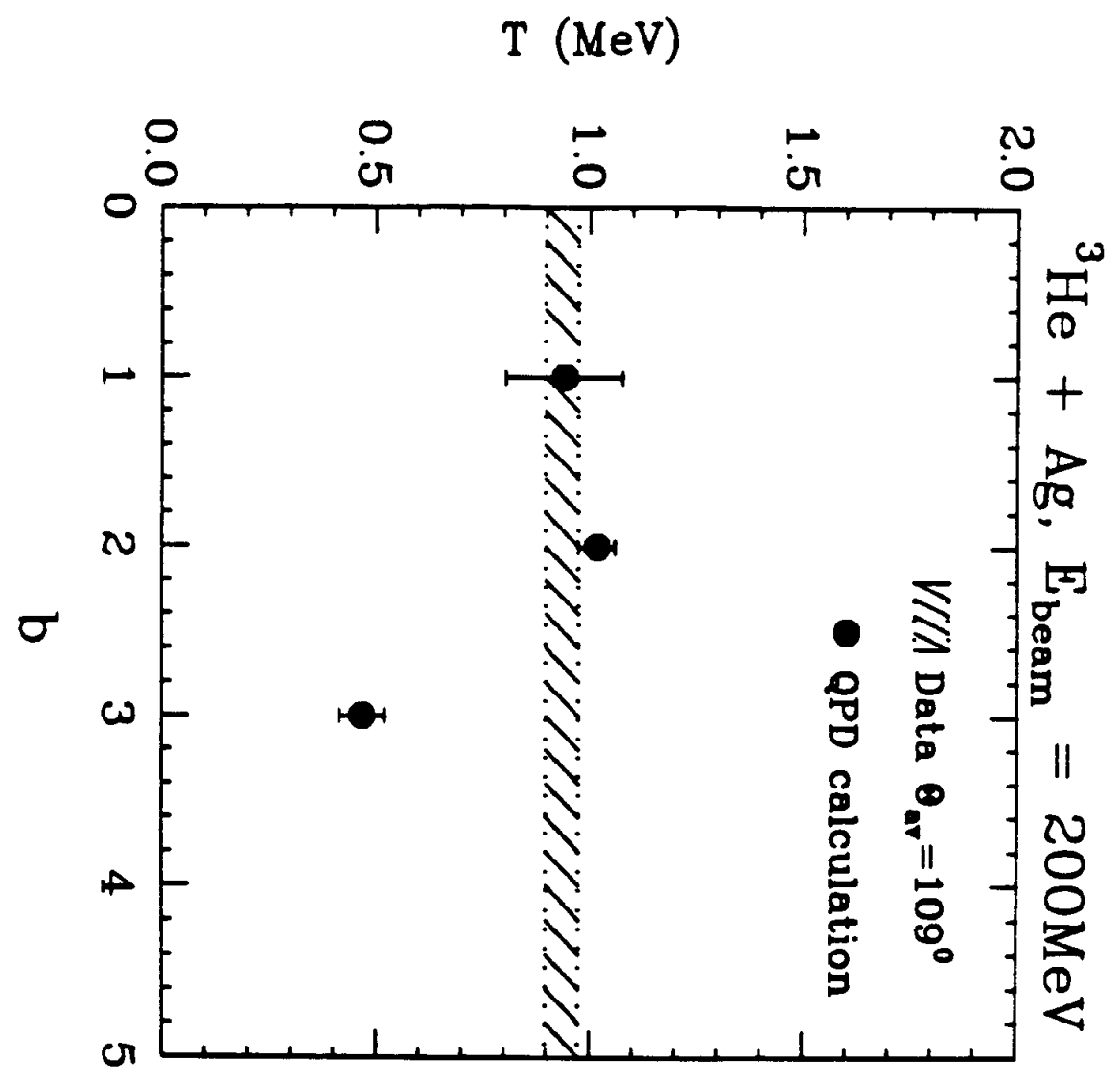

Fig. 4 